\title{
Effects of spin-elastic interactions in frustrated Heisenberg antiferromagnets
}

\author{
Yu. Gaididei \\ Bogolyubov Institute for Theoretical Physics, 252143 Kiev, Ukraine \\ H. Büttner \\ Physikalishes Institut, Universität Bayreuth, D95440 Bayreuth, Germany
}

\begin{abstract}
The Heisenberg antiferromagnet on a compressible triangular lattice in the spin-wave approximation is considered. It is shown that the interaction between quantum fluctuations and elastic degrees of freedom stabilize the low symmetric L-phase with a collinear Néel magnetic ordering. Multi-stability in the dependence of the on-site magnetization is on an uniaxial pressure is found.
\end{abstract}

There is a long standing interest in properties of frustrated quantum antiferromagnets because they display a large variety of behavior: e.g. conventional spin ordering, spin liquid ground states, or chirality transitions [i]. The antiferromagnetic Heisenberg model on a triangular lattice is the simplest two-dimensional frustrated system. Quasi-two-dimensional triangular lattice antiferromagnets are not rare objects. It was recently shown [2] 3] that the materials $A M\left(S_{4}\right)_{2}$ belonging to the Yavapaiite family may be considered as realizations of a quasitwo-dimensional triangular lattice quantum (e.g. $\mathrm{M}=\mathrm{Ti}$ with $\mathrm{S}=1 / 2$ ) and quasiclassical (e.g. $\mathrm{M}=\mathrm{Fe}$ with $\mathrm{S}=5 / 2$ ) antiferromagnets. The rhombohedral $\beta$-phase and monoclinic $\alpha$-phase of solid oxygen consist of weakly interacting planes (triangular in the case of $\beta-\mathrm{O}_{2}$ and rectangular in the case of $\alpha-O_{2}$ (5n) whose magnetic properties are described by the easy-plane Heisenberg antiferromagnetic model with spin $S=1$ [ [1].

Unconventional properties of the $\kappa-(B E D T-$ $T T F)_{2} X$ family can be described by a Hubbard model on a triangular lattice with an anisotropic interactions [6]. An antiferromagnetic Heisenberg model was also used in [7] to describe the properties of a triangular monolayer of $\mathrm{Pb}$ and $\mathrm{Sn}$ adatoms on the (111) surface of Ge form.

From the theoretical point of view the antiferromagnetic Heisenberg model on a triangular lattice has attracted particular attention since Anderson's proposal of a resonance-valence-bond state for this model [8]. From that time much work has been done to understand the nature of its ground state. There is now an almost consensus based on variety of methods 9 12 that the ground state has a conventional three-sublattice order but the situation is close to marginal and quantum fluctuations are important 13]. Quite recently the effect of quantum spin fluctuations on the ground-state properties of spatially anisotropic Heisenberg antiferromagnet in the linear spin-wave approximation was explored [14 16]. The staggered magnetization and the magnon dispersion were calculated as functions of the ratio of the antiferromagnetic exchange between the second and first neighbors, $J_{2} / J_{1}$. As a physical tool which allows to tune the ratio $J_{2} / J_{1}$ Merino et al [16] invoke an uniaxial stress within a layer whereby the relative distances between atoms are changed.

In this paper we investigate the ground-state properties of the compressible frustrated antiferromagnets, i.e. those systems where the coupling between magnetic and elastic degrees of freedom is taken into account,under the action of uniaxial pressure. We treat the system selfconsistently considering the spin subsystem in the linear spin-wave approximation and using the continuum approach for the elastic degrees of freedom. We show that as a result of this method the behavior of the on-site magnetization as a function of the pressure differs significantly from the behavior which was obtained in the framework of the spatially anisotropic Heisenberg model [15, 16. In particular, the on-site-magnetization does not vanish in the whole interval of stability of the collinear phase.

We consider the two-dimensional triangular lattice. The Hamiltonian of the spin subsystem, interacting with the displacements of the magnetic atoms, is

$$
H=\frac{1}{2} \sum_{\vec{n}, \vec{a}} J_{\vec{a}} \vec{S}_{\vec{n}} \vec{S}_{\vec{n}+\vec{a}}
$$

Here $\vec{S}_{\vec{n}}$ is the spin operator of the atom located in the $\vec{n}$-th site of the triangular lattice: $\vec{n}=n_{1} \vec{c}_{1}+$ $n_{2} \vec{c}_{2}\left(n_{1}, n_{2}=0, \pm 1, \pm 2, ..\right)$ where $\vec{c}_{1}=(1,0), \vec{c}_{2}=$ $\left(\frac{1}{2}, \frac{\sqrt{3}}{2}\right)$ are the basic vectors of the triangular lattice, $J_{\vec{a}}=J\left(1-\eta \vec{a}\left(\vec{u}_{\vec{n}+\vec{a}}-\vec{u}_{\vec{n}}\right)\right)=J\left(1-\eta \sum_{i, j} a_{i} u_{i j} a_{j}\right)$ is the exchange integral $(J>0$ is the exchange constant), $\vec{u}_{\vec{n}}$ are the atom displacements from their equilibrium (without magnetic interaction), and $\vec{a}$ is the vector which connects a site with nearest neighbors, $\eta=$ $-d \ln J / d|\vec{a}|$. We consider a small spatially smooth deformation. Therefore it was convenient to introduce the components of the strain tensor $u_{i j}$ : $\quad\left(\vec{u}_{\vec{n}+\vec{a}}-\vec{u}_{\vec{n}}\right)_{i}=$ $\frac{1}{2} \sum_{j}\left(\frac{\partial u_{i}}{\partial n_{j}}+\frac{\partial u_{j}}{\partial n_{i}}\right) a_{j} \equiv \sum_{j} u_{i j} a_{j}, \quad(i, j=x, y)$.

The elastic energy of the two-dimensional triangular lattice in the continuum approximation coincides with the elastic energy of the isotropic plane: $\Phi=\frac{1}{2} K\left(u_{x x}+\right.$ $\left.u_{y y}\right)^{2}+\frac{1}{2} \mu\left(\left(u_{x x}-u_{y y}\right)^{2}+4 u_{x y}^{2}\right)-p_{x} u_{x x}-p_{y} u_{y y}$ where $K$ and $\mu$ are the elastic modulus of the system and $p_{i}(i=x, y)$ are the component of the uniaxial pressure.

Let us use the transformation (see e.g. [17) to the local frame of reference $S_{\vec{n}}^{x}=\tilde{S}_{\vec{n}}^{x} \cos \vec{q} \vec{n}+\tilde{S}_{\vec{n}}^{z} \sin \vec{q} \vec{n}, S_{\vec{n}}^{z}=$ $\tilde{S}_{\vec{n}}^{z} \cos \vec{q} \vec{n}-\tilde{S}_{\vec{n}}^{x} \sin \vec{q} \vec{n}, S_{\vec{n}}^{y}=\tilde{S}_{\vec{n}}^{y}$ in which quantization axis for the spins at each site coincide with its classical direction $(\cos \vec{q} \vec{n}, 0, \sin \vec{q} \vec{n})$ which is determined by 
the vector $\vec{q}$. By using the Holstein-Primakoff spinrepresentation $\tilde{S}_{\vec{n}}^{x}=\sqrt{S / 2}\left(b_{\vec{n}}^{\dagger}+b_{\vec{n}}\right), \quad \tilde{S}_{\vec{n}}^{y}=i \sqrt{S / 2}\left(b_{\vec{n}}^{\dagger}-\right.$ $\left.b_{\vec{n}}\right), \quad \tilde{S}_{\vec{n}}^{z}=S-b_{\vec{n}}^{\dagger} b_{\vec{n}}$ with $b_{\vec{n}}^{\dagger},\left(b_{\vec{n}}\right)$ being the creation (destruction) Bose-operator of the spin-excitation on the $\vec{n}$-th site and applying the Bogolyubov transformation $b_{\vec{n}}=N^{-1 / 2} \sum e^{-i \vec{k} \vec{n}}\left(\cosh \left(\theta_{\vec{k}}\right) \alpha_{\vec{k}}+\sinh \left(\theta_{\vec{k}}\right) \alpha_{-\vec{k}}^{\dagger}\right)$ for these operators $(\vec{k}$ is the wave vector belonging to the first Brillouin zone and $N$ is the number of atoms in the crystal), the Hamiltonian (11) in the spin-wave approximation can be represented in the form $H=N E_{g r}+$ $\sum_{\vec{k}} \omega_{\vec{k}} \alpha_{\vec{k}}^{\dagger} \alpha_{\text {veck }}$. where

$$
E_{g r}=E_{c l}-\frac{1}{N} \sum_{\vec{k}} \sinh ^{2}\left(\theta_{\vec{k}}\right) \omega_{\vec{k}}
$$

is the ground state energy per atom in the spinwave approximation. The coefficients of the Bogolyubov transformation are given by $\tanh ^{2}\left(\theta_{\vec{k}}\right)=$ $\left(f_{\vec{k}}-g_{\vec{k}}\right)^{2}\left(f_{\vec{k}}+g_{\vec{k}}\right)^{-2}$ where

$$
\begin{array}{r}
f_{\vec{k}}^{2}=S(-2 J(\vec{q})+J(\vec{k}-\vec{q})+J(\vec{k}+\vec{q})), \\
g_{\vec{k}}^{2}=2 S(J(\vec{k})-J(\vec{q}))
\end{array}
$$

with $J(\vec{k})=\frac{1}{2} \sum_{\vec{a}} J_{\vec{a}} \cos \vec{k} \vec{a}$, and

$$
\omega_{\vec{k}}=f_{\vec{k}} g_{\vec{k}}
$$

is the magnon energy. $E_{c l}=S^{2} J(\vec{q})$ is the energy of the spin subsystem per atom in the classical approximation.

The on-site magnetization $M \equiv\left\langle\tilde{S}_{\vec{n}}^{z}\right\rangle=S-\left\langle b_{\vec{n}}^{\dagger} b_{\vec{n}}\right\rangle$ can be also obtained by using the Bogolyubov transformation. As a result we get

$$
M=S-\frac{1}{N} \sum_{\vec{k}} \sinh ^{2}\left(\theta_{\vec{k}}\right) .
$$

Our goal now is to minimize the total ground state energy of the system

$$
\mathcal{F}=\Phi+E_{g r} \equiv E_{g r}+J\left(\frac{\kappa}{2}\left(\epsilon_{1}^{2}+\epsilon_{2}^{2}\right)-p \epsilon_{1}\right)
$$

with respect to the variational parameters which are the components of the deformation tensor $\epsilon_{1}=\eta\left(u_{x x}-\right.$ $\left.u_{y y}\right) / 2, \quad \epsilon_{2}=\eta u_{x y}$ and the vector $\vec{q}$. Here the magnetoelastic constant $\kappa=4 \mu /\left(J \eta^{2}\right) \quad$ characterizes the stiffness of the lattice in terms of the intensity of the spinlattice interaction, $p=\left(p_{x}-p_{y}\right) /(\eta J)$ is the dimensionless uniaxial pressure. In Eq. (6) we omitted the terms of spin-lattice interaction which correspond to the dilatation (contraction) of the lattice $\left(\left(u_{x x}+u_{y y}\right) / 2\right)$ without changing its symmetry. These terms don't change the qualitative behavior of the system. The equations for $\vec{q}$ and $\epsilon_{i}$ have the form

$$
\frac{\partial}{\partial \vec{q}} \mathcal{F}=0, \quad \frac{\partial}{\partial \epsilon_{i}} \mathcal{F}=0
$$

Let us consider first the case zero pressure: $p=0$. In the classical approximation when $S \rightarrow \infty$ the energy of the system can be represented in the form

$$
\frac{\mathcal{F}_{c l}}{J}=S^{2} J(\vec{q})+\frac{\kappa}{2}\left(\epsilon_{1}^{2}+\epsilon_{2}^{2}\right)
$$

¿From Eq.(8) we obtain that the set of equations (7) has three types of solutions:

i) H-phase: $\vec{q}_{H}=4 \pi / 3(\cos \phi, \sin \phi), \quad \phi=0, \pm \pi / 3$. The spin structure corresponding to each $\vec{q}_{H}$ is a threesublattice antiferromaget.Three different $\vec{q}_{H}$ represent three possible antiferromagnetic domains. The lattice structure is an undistorted triangular lattice $\left(\epsilon_{1}=\epsilon_{2}=\right.$ 0) with the group symmetry $D_{6 h}$.

ii) L-phase: $\vec{q}_{L}=2 \pi / \sqrt{3}(\sin \phi, \cos \phi), \quad \phi=0, \pm \pi / 3$. The spin structure corresponding to each $\vec{q}_{L}$ is a twosublattice antiferromagnet. The lattice structure for $\phi=0$ is determined by the deformation tensors $\epsilon_{1}=$ $2 S^{2} / \kappa, \epsilon_{2}=0$ while for two other $\vec{q}_{L}$ the deformation tensors can be obtained by rotations by $\pm \pi / 3$. The lattice is characterized by the group symmetry $D_{2 h}$.

iii) S-phase: $\vec{q}_{s}=\left(q_{s}, 2 \pi / \sqrt{3}\right)$, $\epsilon_{1}=\kappa^{-1}\left(\cos q_{s}+\cos \left(q_{s} / 2\right)\right), \epsilon_{2}=0$ where $\cos \left(q_{s} / 2\right)=$ $\left(\sqrt{9+32 \kappa / S^{2}}-5\right) / 8$. Two more vectors $\vec{q}$ and deformation tensors $\epsilon_{1}$ and $\epsilon_{2}$ can be obtained from the above equation by rotations by $\pm \pi / 3$. The spin structure is a spiral antiferromagnet.

We consider the stability of obtained solutions for the case when

$$
\epsilon_{1} \geq 0, \quad \epsilon_{2}=0, \quad 0 \leq q_{x} \leq \frac{2 \pi}{3}, \quad q_{y}=\frac{2 \pi}{\sqrt{3}},
$$

In the subspace (9) the stability condition reads

$$
\frac{\partial^{2} \mathcal{F}}{\partial \epsilon_{1}^{2}}>0, \quad \frac{\partial^{2} \mathcal{F}}{\partial \epsilon_{1}^{2}} \frac{\partial^{2} \mathcal{F}}{\partial q_{x}^{2}}-\left(\frac{\partial^{2} \mathcal{F}}{\partial q_{x} \partial \epsilon_{1}}\right)^{2}>0
$$

One can obtain from Eqs (8) and (10) that

- the $L$-phase is stable when $\kappa / S^{2}<\frac{9}{4}$

- both $H$ - and $L$-phases are stable when $\frac{9}{4}<\kappa / S^{2}<$ 5 . For $\kappa / S^{2}=4$ the $\mathrm{H}$ - and L-phases have the same energy while for $\kappa / S^{2}<4$ the L-phase becomes energetically more favorable.

- the $H$-phase is stable when $\kappa / S^{2}>5$.

The spiral S-phase does not correspond to a minimum of the ground state energy. In the subspace (9) the spiral phase for $\frac{9}{4}<\kappa / S^{2}<5$ corresponds to the saddle point which separates two stable phases $H$ and $L$.

The wave vectors $\vec{q}_{H}$ and $\vec{q}_{L}$ determine also the magnetic structure of the $\mathrm{H}$ - and L-phases in the spin-wave approximation. This is not true, however, for the spiral 
phase where quantum fluctuations change the value of the spiral wave-vector $q_{s}$.

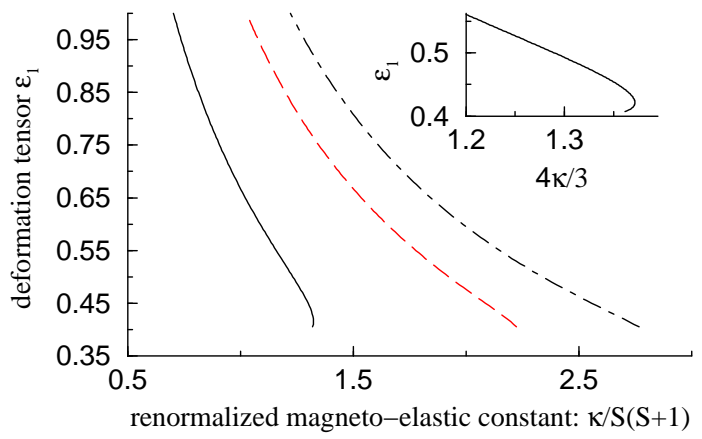

FIG. 1. The deformation tensor $\epsilon_{1}=\left(u_{x x}-u_{y y}\right) / 2$ for the collinear L-phase (full curve $S=1 / 2$, dashed curve $S=1$, dotted-dashed curve $\mathrm{S}=3 / 2$ ) as a function of the renormalized magneto-elastic constant $\kappa / S(S+1)$. Multistability for the case $S=1 / 2$ is shown in the inset.

Evaluating numerically the integrals in the r.h.s. of Eq. (2) for $\vec{q}=\vec{q}_{H}, \epsilon_{1}=0$ one can obtain that the ground state energy of the symmetric triangular phase in the spin-wave approximation is $\mathcal{F}_{H} \approx$ $J\left(-\frac{3}{2} S(S+1)+1.1723 S\right)$. We have also found that the $\mathrm{H}$-phase is stable for $\kappa>\kappa_{H}$ where

$$
\frac{\kappa_{H}}{S^{2}}= \begin{cases}6.86, & \text { for } S=\frac{1}{2} \\ 3.85, & \text { for } S=1 \\ 3.26, & \text { for } S=\frac{3}{2} \\ 2.99, & \text { for } S=2\end{cases}
$$

Considering the low-symmetry L-phase which corresponds to the wave vector $\vec{q}_{L}=2 \pi(0,1 / \sqrt{3})$ we obtain from Eqs (3), (4) that its energy in the spin-wave approximation is determined by the expression

$$
\begin{array}{r}
\frac{\mathcal{F}_{L}}{J}=-S(S+1)\left(1+2 \epsilon_{1}\right)+\frac{\kappa \epsilon_{1}^{2}}{2}+S I\left(\epsilon_{1}\right) \\
I\left(\epsilon_{1}\right)=\left(2+\epsilon_{1}\right) \frac{4}{\pi^{2}} \int_{0}^{\frac{\pi}{2}} d y\left(1-2 \frac{1-\epsilon_{1}}{2+\epsilon_{1}} \sin ^{2} y\right) E(m)
\end{array}
$$

where $E(m)$ is the elliptic integral of the second kind [19] with the parameter $m=\cos ^{2} y\left(1-2 \frac{1-\epsilon_{1}}{2+\epsilon_{1}} \sin ^{2} y\right)^{-2}$. One can obtain from Eq. (4) that in the $\vec{k} \rightarrow 0$ limit the magnon dispersion in the L-phase has the form $\omega_{L}(\vec{k})=$ $2 J S\left(2+\epsilon_{1}\right) \sqrt{\left(5 \epsilon_{1}-2\right) k_{x}^{2}+3\left(2+\epsilon_{1}\right) k_{y}^{2}}$. This means that the magnons in the low-symmetry L-phase can exist only for $\epsilon_{1}>0.4$ and only in this interval we may look for extrema of the function $\mathcal{F}_{L}$. It is seen from Eqs (11) that the quantity $J_{1}=J\left(1+\epsilon_{1} / 2\right)\left(J_{2}=J\left(1-\epsilon_{1}\right)\right)$ corresponds to the antiferromagnetic exchange between first (second) neighbors in the spin-wave theory of Heisenberg antiferromagnet on an anisotropic triangular lattice [15,16. Therefore the interval $1>\epsilon_{1}>0.4$ corresponds to the interval $0<J_{2} / J_{1}<1 / 2$ in the spin-wave theory of Heisenberg antiferromagnet on an anisotropic triangular lattice.

In Fig. 11 we plot the deformation tensor $\epsilon_{1}$ which provides an extremum of $\mathcal{F}_{L}$, as a function of the coupling parameter $\kappa$ for three spin values $S=1 / 2,1,3 / 2$. It is seen that the equation $d \mathcal{F} / d \epsilon_{1}=0$ has solution $\epsilon_{1}$ only for $\kappa<\kappa_{L}$. The critical value $\kappa_{L}$ which determines the interval of the existence of the L-phase depends on the spin $S$. It increases when the spin $S$ increases, e.g.

$$
\frac{\kappa_{L}}{S^{2}}= \begin{cases}3.97, & \text { for } S=\frac{1}{2} \\ 4.47, & \text { for } S=1 \\ 4.64, & \text { for } S=\frac{3}{2} \\ 4.73, & \text { for } S=2\end{cases}
$$

but even for $S=4$ it is still less $\left(\kappa_{L} / S^{2} \approx 4.75\right)$ than the critical value for this parameter obtained in the classical approximation $\left(\kappa_{L} / S^{2}=5\right)$. The reason for this is the strong contribution to the effective elastic energy of the system from quantum fluctuations (the second term in the r.h.s. of Eq. (12)).The quantum fluctuations change both the stiffness of the lattice (the second derivative of the free energy (12) with respect to the deformation $\epsilon_{1}$ ) and the constant of the spin-elastic interaction ( the first derivative of the free energy). A single-valued monotonic dependence $\epsilon_{1}(\kappa)$ is obtained for $S \geq 1$. For $S=1 / 2$ the dependence becomes multi-valued, i.e. there is an interval of $\kappa$ where two values $\epsilon_{1}^{(1)}$ and $\epsilon_{1}^{(2)}\left(\epsilon_{1}^{(1)}<\epsilon_{1}^{(2)}\right)$ of the deformation tensor correspond to each value of the coupling constant $\kappa$. Only the solution which corresponds to the deformation $\epsilon_{1}^{(2)}$ corresponds to the minimum of the effective elastic energy (12).

To find the stability region of the collinear L-phase we numerically evaluated integrals in the l.h.s. of the inequality (10) and found that it holds in the interval $\kappa<\kappa_{L}$. Comparing the stability limits for $\mathrm{H}$ - and Lphases give in Eqs (11) and (13) we find the following interesting results. For $S \geq 1$ we have always $\kappa_{H}<\kappa_{L}$ like in the classical approach where the stability regions for H- and L-phases overlap. However, for $S=1 / 2$ this is not the case since there is an interval $\kappa_{L}<\kappa<\kappa_{H}$ where neither the L- nor the H-phase exist.

The nature of the phase in the interval $\left(\kappa_{L}, \kappa_{H}\right)$ or in other words for $\epsilon_{1}<0.4$, cannot be clarified in the framework of the spin-wave approach. The reason why this approach fails is the following. As it was mentioned above the vectors $\vec{q}_{s}$ obtained in the classical approach are not solutions of the extrema conditions (7) in the spin-wave approximation. On the other hand, as it is seen from Eqs (4) and (3) the magnon frequency $\omega_{\vec{k}}$ is real in the interval $\epsilon_{1}<0.4$ only for $\vec{q}$ obtained in the classical approach. For other $\vec{q}$ values $\omega_{\vec{k}}^{2}$ is not positive definite. Thus to check the stability of the corresponding phase is stable one should calculate the magnon dispersion beyond the spin-wave approach. 


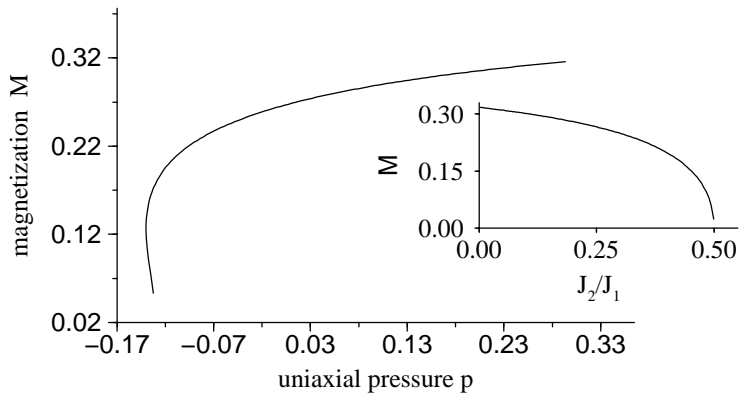

FIG. 2. The effect of the uniaxial pressure on the on-site magnetization in the L-phase $(S=1 / 2, \kappa=1.315)$. The inset represents the magnetization as a function of the ratio $J_{2} / J_{1}=2\left(1-\epsilon_{1}\right) /\left(2+\epsilon_{1}\right)$ of the effective exchange integrals between the second and first neighbors in the presence of the uniaxial pressure.

Let us consider how the L-phase evolves in the presence of an uniaxial pressure $p$. From Eqs (6) and (7) we get

$$
\begin{array}{r}
\vec{q}=\frac{2 \pi}{\sqrt{3}}(0,1), \quad \epsilon_{2}=0, \\
p=\kappa \epsilon_{1}-2 S(S+1)+S \frac{d I\left(\epsilon_{1}\right)}{d \epsilon_{1}}
\end{array}
$$

In Fig. 2 we show results for the on-site magnetization in the L-phase for $S=\frac{1}{2}$ as a function of the uniaxial pressure $p$. Fig. 2 and in particular its inset shows that our results are qualitatively different from those of a recent spatially anisotropic triangular lattice study 15, 16. In contrast to the $\left(J_{1}, J_{2}\right)$-model [15, 16 where the ratio $J_{2} / J_{1}$ was a free parameter in our model the corresponding parameter in a compressible triangular lattice $J_{2} / J_{1}=2\left(1-\epsilon_{1}\right) /\left(2+\epsilon_{1}\right)$ is determined selfconsistently from the extrema conditions (7). It is seen that in the interval of the existence of the L-phase we have $J_{2} / J_{1}<1 / 2 \quad\left(\epsilon_{1}>0.4\right)$ and the on-site magnetization is finite. It is interesting that there exists an interval where two values of the magnetization $M$ correspond to each value of the pressure $p$. One can show, however, that only the state with a larger value of $M$ is stable.

We conclude that the Heisenberg antiferromagnet on an compressible triangular lattice differs qualitatively from the Heisenberg antiferromagnet on an anisotropic triangular lattice. Self-consistent treatment of the elastic degrees of freedom shows that the spiral magnetic phase is unstable in the classical approximation. This approach shows also that in the spin-wave approximation the interaction between quantum fluctuations and elastic degrees of freedom stabilizes the collinear L-phase.

Acknowledgements

Yu. Gaididei is grateful for the hospitality of the University of Bayreuth

where this work was performed. Partial support from the DRL grant Nr.: UKR-002-99 is also acknowledged.

[1] Magnetic Systems with Competing Interactions, edited by H.T. Diep (World Scientific, Singapore, 1994).

[2] S.T. Bramwell, S.G. Carling, C.J. Harding, K.D.M. Harris, B.M. Kariuki, L. Nixon, and I.P. Parkin, J.Phys.: Condens. Matter. 8, L123 (1996).

[3] H. Serrano-González, S.T. Bramwell, K.D.M. Harris, B.M. Kariuki, L. Nixon, I.P. Parkin, and C. Ritter, Phys. Rev. B 59, 14451 (1999).

[4] Yu.B. Gaididei, V.M. Loktev, A.F. Prikhotko, and L.I. Shansky, Sov. J. Low Temp. Phys. 1,653 (1975) (Fiz. Nizk. Temp. 1, 1365 (1975)).

[5] I. N. Krupskii, A. I. Prokhvatilov, Yu. A. Freiman, and A. I. Erenburg, Sov. J. Low Temp. Phys. ,130 (1979) (Fiz. Nizk. Temp. 5, 271 (1979)).

[6] R.H. McKenzie, Comment. Condens. Matter Phys. 18, 309 (1998).

[7] J.P. Rodriguez and E. Artacho, Phys.Rev. B 59, R705 (1999).

[8] P.W. Anderson, Mater. Res. Bull. 8, 153 (1973); P.Fazekas and P.W. Anderson, Philos. Mag. 30, 423 (1974).

[9] D.A. Huse and V. Elser, Phys.Rev.Lett. 60,2531 (1988).

[10] Th. Jolicoeur and J.C. Le Guillou, Phys. Rev. B 40,2727 (1989).

[11] R. Deutscher, H.U. Everts, S. Miyashita, and J. Wintel, JPhys. A 23, L1043 (1993).

[12] L. Caprioti, A. E. Trumper, and S. Sorella, Phys. Rev. Lett. 82, 3899 (1999).

[13] P.W. Leung and K.J. Runge, Phys.Rev. B 58, 73 (1993).

[14] U. Bhaumik and I. Bose, Phys. rev. B 58, 73 (1998).

[15] A. E. Trumper, Phys. Rev. B 60, 2987 (1999).

[16] J. Merino, R.H. McKenzie, J.B. Marston, and C.H. Chung, J. Phys.:Condens. Matter 11, 2965 (1999).

[17] S.V. Tyablikov, Metody Kvantovoi Teorii Magnetizma (Nauka, Moskva, 1975).

[18] Yu. B. Gaididei, V. M. Loktev, Sov. J. Low.Temp. Phys. 7, 634 (1981)(Fiz. Nizk. Temp. 7,1305 (1981)).

[19] M.Abramowitz and I.Stegun, Handbook of Mathematical Functions (Dover Publications,Inc.,New York,1972) 